\title{
Platelet-rich plasma versus corticosteroid injections for rotator cuff tendinopathy: a comparative study with up to 18-month follow-up
}

\author{
Juho Aleksi Annaniemi ${ }^{1,2}$, Jüri Pere ${ }^{1}$, Salvatore Giordano ${ }^{3}$ \\ ${ }^{1}$ Department of Surgery, Welfare District of Forssa, University of Turku, Forssa, Finland \\ ${ }^{2}$ Department of Orthopedics and Traumatology, Turku University Hospital, University of Turku, Turku, Finland \\ ${ }^{3}$ Department of General and Plastic Surgery, Turku University Hospital, University of Turku, Turku, Finland
}

\begin{abstract}
Background: Given the complications involved in corticosteroid (CS) injections, subacromial platelet-rich plasma (PRP) injections may provide a valid alternative to CS in the treatment of rotator cuff (RC) tendinopathy.

Methods: We retrospectively reviewed a total of 98 patients affected by RC tendinopathy who were treated with either subacromial injection of PRP or CS. The PRP group received three injections of autologous PRP at 2 weeks interval, and the CS group received one injection of CS. The Western Ontario Rotator Cuff Index (WORC) was the primary outcome measure, while the secondary outcome measures were the visual analog scale (VAS), range of motion (ROM), and need for cuff repair surgery, which were analyzed at intervals of 6,12 , and 18 months.

Results: A total of 75 patients were included in the analysis (PRP, $n=35$; CS, $n=40$ ). The mean follow-up for PRP was $21.1 \pm 8.7$ months and for CS was 33.6 \pm 16.3 months ( $<<0.001$ ). Both groups showed improvement in WORC, VAS, and ROM. No significant differences were detected between the two groups in any of the primary (WORC) or secondary outcomes over 6,12 , and 18 months (all p $>0.05$ ). No adverse events were detected.

Conclusions: Both treatments improved patient symptoms, but neither resulted in a significantly better outcome in this series of patients. PRP can be a safe and feasible alternative to CS, even at long-term follow-up, to reduce local and systemic effects involved with CS injections.
\end{abstract}

Keywords: Platelet-rich plasma; Adrenal cortex hormones; Injections; Rotator cuff; Tendinopathy; Shoulder pain

\section{INTRODUCTION}

An estimated $0.9 \%$ to $2.5 \%$ of the population reports shoulder area pain, and the prevalence increases rapidly with age, reaching as high as $6.7 \%$ to $66.7 \%$ over a lifetime [1]. Shoulder area pain is often complex and multifaceted and may be accompanied by several changes in the shoulder structures [2]. Rotator cuff (RC) tendinopathy is the degeneration of the four RC muscle tendons, and calcific deposits of the tendon can be concurrent [2,3]. General guidelines suggest that tendinopathy should be treated pri-

Received: September 9, $2021 \quad$ Revised: October 19, $2021 \quad$ Accepted: October 26, 2021

Correspondence to: Salvatore Giordano

Department of General and Plastic Surgery, Turku University Hospital, University of Turku, Savitehtaankatu 1, Turku 20521, Finland

Tel: +358-443346119, Fax: +358-2-3132284, E-mail: salvatore.giordano@tyks.fi, ORCID: https://orcid.org/0000-0001-5981-6870

\section{Financial support: None.}

Conflict of interest: None. 
marily non-operatively $[3,4]$. Treatment options include various injection therapies, analgesic drugs, physical therapy, extracorporeal shockwave therapy, and ultrasound guided needling (barbotage) [2,3].

Injection therapy options include corticosteroids (CSs), platelet-rich plasma (PRP), hyaluronic acid, and botulinum toxin [5]. A meta-analysis suggests that CS may yield short-term symptom alleviation; however, PRP and prolotherapy may be better in the long term [5]. PRP injection therapies have shown great potential in RC-related problems as well as in other tendon and joint related disorders [6,7].

Previous in vivo and in vitro studies suggest that CS injections may reduce symptoms in tendinopathies but may also cause systemic disorders and further damage to the soft tissue. CS injections reduce cellular proliferation, affect collagen and extracellular matrix composition, do not permit inflammatory pathways, and increase adipocyte differentiation and cellular apoptosis [811]. Furthermore, these changes can be detected after only 24 hours and last 2 to 3 weeks, resulting in reducing maximal load to failure and tendon stiffness. Despite that, shoulder subacromial CS injections are commonly performed.

There is evidence that analgesic substances commonly used concurrently with CS injections may cause unfavorable effects in the soft tissue structures [12]. In contrast, PRP injections have not been shown to have any significant negative effects or adverse effects, suggesting that PRP may have a multitude of beneficial effects regarding soft tissue healing [13,14]. Only few studies directly compared subacromial injections of PRP with CS, and further comparisons to the widely used CS injections are still warranted. Most previous studies were conducted using a very small number of patients and/or had a short follow-up [6,13,15-19]. Despite the encouraging results of PRP injections used concurrently with arthroscopic RC repair, there is still little information about its effects solely as an injection therapy $[6,20,21]$. There is currently limited understanding how PRP compares to other conservative treatment options, and a meta-analysis suggests that the results of the previous studies are to be interpreted with caution [6].

The aim of this study was to compare the clinical outcomes of subacromial PRP and CS injections in RC tendinopathy in terms of symptoms relief and functional improvement. We hypothesized that subacromial PRP injections may yield equal results to CS injections in RC tendinopathy treatment.

\section{METHODS}

This study was approved by the Institutional Review Board (39/ 13.01.01/2018, Welfare District of Forssa, Finland), and the pres- ent study was conducted in accordance with the ethical principles of the World Medical Association Declaration of Helsinki. Individual informed consent was waived by the local Ethics Committee of the University of Turku due to the retrospective nature of the study and the de-identification of the source data. This is a single-centered retrospective study. We reviewed 98 consecutive patients with RC and shoulder disorders who underwent subacromial PRP injections or a single CS injection between 2014 and 2018 at the Department of Surgery of the Welfare District of Forssa, Finland To compare the outcomes of these two treatment modalities, patients were divided into two groups.

Patients received either single injection of $2 \mathrm{~mL}(40 \mathrm{mg} / \mathrm{mL})$ methylprednisolone acetate (Solomet, Orion, Espoo, Finland; Depo-Medrol, Pfizer, New York, NY, USA) or three 1-2 mL injections of autologous PRP (Commercial Glo PRP kit; Glofinn Oy, Salo, Finland) in the subacromial space. The injection was performed by an experienced orthopedist using anatomical landmarks without ultrasound guidance.

The PRP preparation protocol was as follows: $9 \mathrm{~mL}$ of venous blood was drawn from the patient, and a red blood cell (RBC) collector was connected to the syringe. The blood was centrifuged at 1,200 revolutions per minute (rpm) for 5 minutes, excess RBCs were discarded, and a second centrifugation was performed for 10 minutes at 1,200 rpm. White blood cells were not separated from the PRP. The final product contained approximately 1-2 mL of PRP with four to eight times higher platelet concentration than the normal physiological level. PRP injections were given at 2 weeks intervals.

Following diagnosis, all patients were instructed by a physical therapist to exercise their shoulder using a pendulum motion and finger wall climbing with the upper extremity as routine protocol for patients with RC tendinopathy.

Inclusion criteria were age between 18 and 90 years, diagnosed RC tendinopathy, other causes ruled out by imaging and clinical inspection, and a preintervention visual analog scale (VAS) of 30-100. Diagnosis and ruling out of other disorders not fitting to be RC tendinopathy were conducted using X-ray imaging and magnetic resonance imaging (MRI) and/or ultrasound as well as clinical inspection by an experienced orthopedist. In the study, we included small partial tears or intratendinous tears that showed clear degenerative origin. Exclusion criteria were traumatic RC ruptures, full thickness RC ruptures, fractures, frozen shoulder, nerve-related symptoms, labral and long tendon of the biceps muscle tears, and osteoarthritis of the acromion-clavicular joint and glenoid-humeral joint. General conditions requiring surgical intervention as a primary care were excluded; therefore, patients who underwent any shoulder 
surgical procedure during the study time were excluded from the follow-up count. We also excluded patients with major systemic disorders (e.g., hematological diseases, infections, immunodeficiency), pregnancy or possible pregnancy, and patients who received any other kind of subacromial injections or oral medication other than paracetamol or nonsteroidal anti-inflammatory drugs (NSAIDs). Electronic medical records showed that all patients used either paracetamol or NSAID (typically ibuprofen) medication prescribed for pain to be taken when needed. The maximum dosage of paracetamol was 3,000 mg per day, and the maximum dosage of ibuprofen was of $1,800 \mathrm{mg}$ per day.

Patient demographics, treatment modality, frequency of treatment, imaging results, and clinical outcomes were carefully collected both from the patients' electronic medical records as well as the prospectively maintained departmental database. The primary outcome measurement was the Western Ontario Rotator Cuff Index (WORC), and secondary outcome measures were the VAS, range of motion (ROM), and need for surgical intervention. The parameters were recorded before the treatments and at 6, 12, and 18 months, or more. Typical findings in the patients' MRI scans were tendinosis/tendinitis in the tendon of supraspinatus muscle with or without subacromial bursitis and in some cases tendinosis/tendinitis of other RC muscle (infraspinatus, teres minor or subscapularis). One patient in the CS group also had subscapularis and infraspinatus tendinitis/tendinosis with edema but without supraspinatus tendinosis. The PRP group included one patient with a minor intra-tendinous rupture of the supraspinatus tendon with accompanying tendinosis. Tendon swelling varied within both groups from minor swelling to clearly increased swelling in the MRI scans. Roughly 33 patients had signs of calcific deposits in their RC tendons as part of the tendinopathy. This swelling, calcific deposits, and edema in the surrounding structures, namely in the subacromial bursa, resulted in an equally varying degree of impingement in the MRI.

The patient selection protocol and follow-up points are described in Fig. 1. A total of 75 patients (PRP, $n=35$; CS, $n=40$ ) were included in the final analysis after inclusion and exclusion criteria were applied.

We showed the measures of parametric and nonparametric as mean \pm standard deviation. Statistical analyses were carried out using IBM SPSS ver. 23.0 (IBM Corp., Armonk, NY, USA). A two-sided $p$-value of $\leq 0.05$ was set as statistically significant. For comparisons between the study groups, we used Student t-test for continuous variables and Fisher exact test for discrete variables, according to the data type. We calculated the post hoc statistical power of $47.5 \%$ concerning the primary outcome measure including an observed effect size of 0.436 (Cohen's d).

\section{RESULTS}

A total of 75 patients treated for RC tendinopathy between 2014 and 2018 were included in the final analysis. Of them, 35 patients (47\%) received PRP injections while 40 (53\%) received a CS injection. Surgery was the most common end point before reaching 18 months of follow-up (Fig. 1). Demographic data are outlined in Fig. 2 and showed a significant difference in the sex ratio (PRP female to male, $28: 7$ vs. CS, $23: 17 ; \mathrm{p}=0.048$ ) and having any comorbidities, which were higher in the CS-group (PRP, 7 [20\%] vs. CS, 19 [47.5\%]; $\mathrm{p}=0.013$ ) (Fig. 2). There were no statistically significant differences between the groups in other demographics data (Fig. 2).

We evaluated the preintervention parameters and identified differences in the WORC emotions subscore (PRP, 189.7 \pm 56.0 vs. CS, $146.7 \pm 74.7 ; \mathrm{p}=0.007$ ), but no other parameters (Table 1 ). The preintervention WORC lifestyle subscore showed a trend towards the PRP group, but no statistical significance was detected

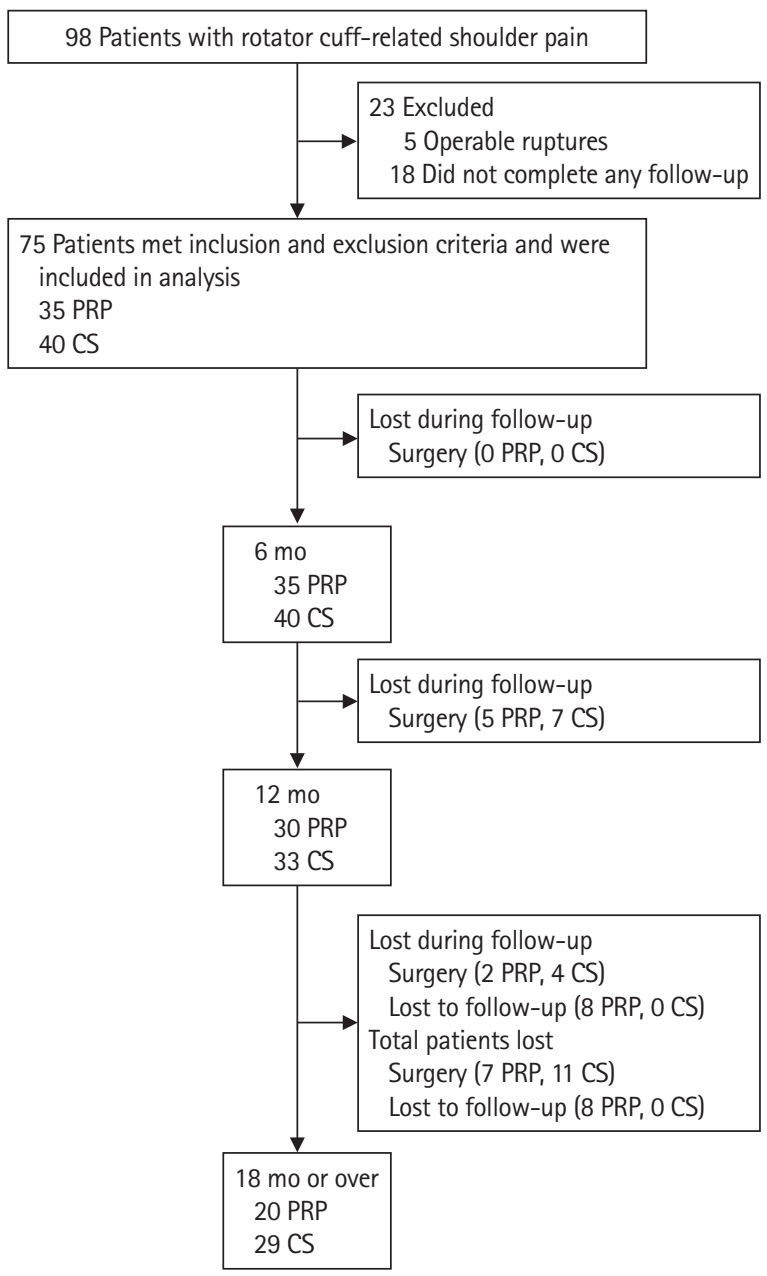

Fig. 1. Patient selection protocol and follow-up flow. PRP: platelet-rich plasma, CS: corticosteroid. 


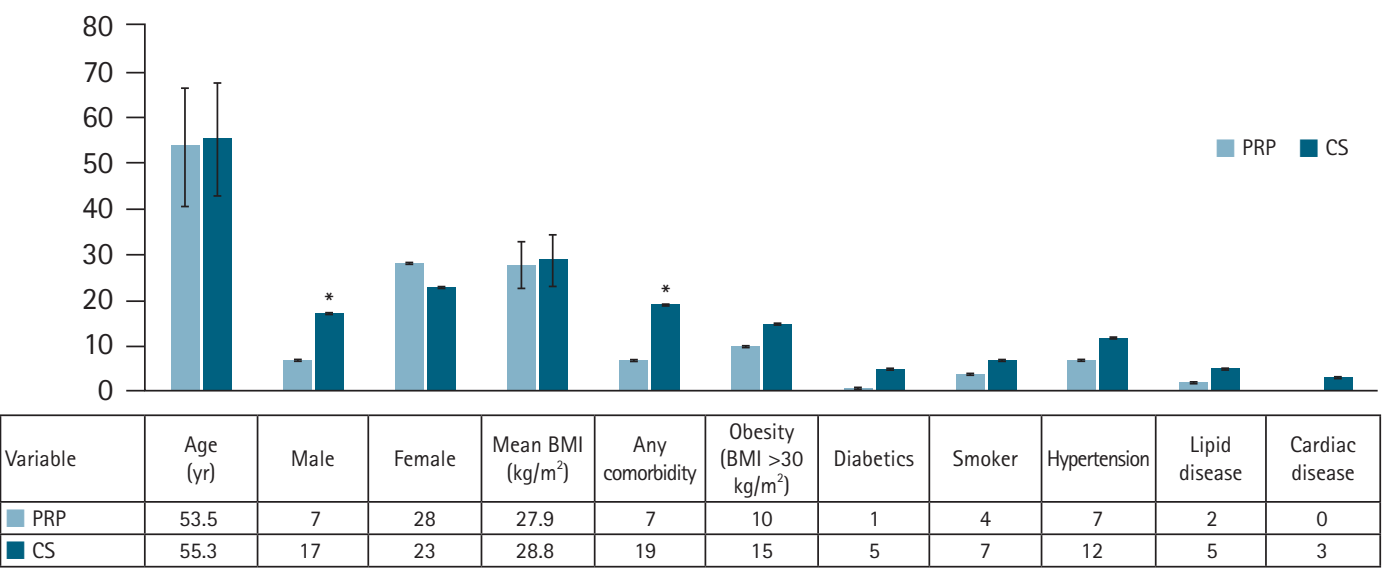

Fig. 2. Demographic data of the patients at the time of study. Age and body mass index (BMI) are presented in mean values with 1 standard deviation (SD), and the rest are absolute numeric values (no mean or SD). PRP: platelet-rich plasma, CS: corticosteroid. ${ }^{\star}$ Significant statistical difference $(\mathrm{p}<0.05)$ between the two groups.

Table 1. Comparison of preinterventional parameters in the two groups of patients

\begin{tabular}{lccc}
\hline Variable & PRP group $(\mathrm{n}=35)$ & CS group $(\mathrm{n}=40)$ & $\mathrm{p}$-value \\
\hline Bilateral & $2(5.7)$ & $2(5.0)$ & 1.000 \\
Visual analog scale score & $70.4 \pm 10.7$ & $69.4 \pm 13.7$ & 0.714 \\
WORC total score & $1331.1 \pm 307.6$ & $1233.1 \pm 270.0$ & 0.146 \\
$\quad$ Physical & $49.2 \pm 14.4$ & $49.5 \pm 13.3$ & 0.921 \\
$\quad$ Sports & $25.3 \pm 18.6$ & $26.4 \pm 14.7$ & 0.786 \\
Work & $30.3 \pm 19.8$ & $32.8 \pm 18.1$ & 0.560 \\
$\quad$ Lifestyle & $253.3 \pm 76.0$ & $222.9 \pm 68.2$ & 0.072 \\
$\quad$ Emotion & $189.7 \pm 56.0$ & $146.7 \pm 74.7$ & $0.007^{\star}$ \\
Range of motion $\left(^{\circ}\right)$ & & & $145.5 \pm 44.0$ \\
$\quad$ Frontal & $138.3 \pm 40.7$ & $121.2 \pm 50.6$ & 0.466 \\
$\quad$ Abduction & $123.6 \pm 46.4$ & & 0.838 \\
\hline
\end{tabular}

Values are presented as number (\%) or mean \pm standard deviation.

PRP: platelet-rich plasma, CS: corticosteroid, WORC: Western Ontario Rotator Cuff Index.

${ }^{\star}$ The significant statistical difference $(\mathrm{p}<0.05)$ between the two groups.

(PRP, $253.3 \pm 76.0$ vs. CS, $222.9 \pm 68.2 ; \mathrm{p}=0.072$ ) (Table 1).

The postinterventional data showed no significant differences in the WORC, ROM, or VAS scores between the two groups at 6, 12, or 18 months (all p >0.05) (Tables 2 and 3), (Figs. 3 and 4). We also detected the number of shoulder surgeries during the follow-up period, but there was no difference between the groups, although there were fewer cases in the PRP group (PRP, 7 [20\%] vs. CS, 11 [27.5\%]; p=0.589) (Table 3).

No adverse events were detected during the follow-up because of the injection procedures. The PRP group had more injections than CS group due to the different treatment protocol. The mean follow-up was over 20 months in both groups, but there was a significant difference between groups favoring patients treated with CS (PRP, $21.1 \pm 8.7$ vs. CS, $33.6 \pm 16.3$ months; $\mathrm{p}<0.001$ ) (Table 2).

\section{DISCUSSION}

Our study demonstrated that PRP is not inferior to CS in any of the measured parameters. Both groups experienced similar benefits from the injection therapies with no statistical differences detected in WORC, ROM, or VAS scores at 6, 12, and 18 months. No adverse effects were detected in either of the two groups. To our knowledge, this is the first study of RC tendinopathy patients treated with either PRP or CS injections showing that PRP is not inferior to CS even in long-term follow-up.

Our results are consistent with current literature, showing that PRP may be a beneficial treatment for RC tendinopathy $[5,17$, 19,22]. Previous studies are controversial in interpreting the efficacy of PRP injections due to the different research and treatment protocols; in many cases, they involve arthroscopy or different products of PRP (for example PRP fibrin matrix) [5,20,21]. We 
Table 2. Comparison of postinterventional parameters in the two groups of patients at 6,12 and 18 months follow-up

\begin{tabular}{|c|c|c|c|}
\hline Variable & PRP group & CS group & p-value \\
\hline 6-Month follow-up & $(n=35)$ & $(\mathrm{n}=40)$ & \\
\hline VAS score & $28.1 \pm 29.3$ & $22.2 \pm 31.4$ & 0.410 \\
\hline WORC total score & $606.3 \pm 612.9$ & $446.3 \pm 570.9$ & 0.224 \\
\hline Physical & $149.4 \pm 146.0$ & $112.2 \pm 142.3$ & 0.274 \\
\hline Sports & $143.3 \pm 143.3$ & $108.2 \pm 131.1$ & 0.278 \\
\hline Work & $129.7 \pm 130.2$ & $94.9 \pm 126.6$ & 0.251 \\
\hline Lifestyle & $107.7 \pm 122.5$ & $76.8 \pm 109.3$ & 0.259 \\
\hline Emotion & $81.1 \pm 96.9$ & $54.7 \pm 83.3$ & 0.141 \\
\hline \multicolumn{4}{|l|}{$\operatorname{ROM}\left(^{\circ}\right)$} \\
\hline Frontal & $161.9 \pm 34.3$ & $168.4 \pm 25.8$ & 0.357 \\
\hline Abduction & $160.4 \pm 36.2$ & $159.9 \pm 35.4$ & 0.948 \\
\hline 12-Month follow-up & $(\mathrm{n}=30)$ & $(\mathrm{n}=33)$ & \\
\hline VAS score & $17.1 \pm 27.6$ & $13.8 \pm 28.2$ & 0.647 \\
\hline WORC total & $372.9 \pm 552.2$ & $285.8 \pm 474.0$ & 0.506 \\
\hline Physical & $99.1 \pm 143.2$ & $75.4 \pm 121.1$ & 0.483 \\
\hline Sports & $84.7 \pm 124.2$ & $74.2 \pm 112.4$ & 0.730 \\
\hline Work & $80.7 \pm 122.2$ & $60.1 \pm 105.4$ & 0.480 \\
\hline Lifestyle & $59.8 \pm 102.8$ & $41.7 \pm 84.1$ & 0.448 \\
\hline Emotion & $48.6 \pm 83.5$ & $34.2 \pm 71.2$ & 0.467 \\
\hline \multicolumn{4}{|l|}{$\operatorname{ROM}\left(^{\circ}\right)$} \\
\hline Frontal & $170.7 \pm 22.8$ & $174.2 \pm 16.6$ & 0.482 \\
\hline Abduction & $168.8 \pm 26.0$ & $168.8 \pm 25.6$ & 0.999 \\
\hline 18-Month follow-up & $(n=20)$ & $(n=29)$ & \\
\hline VAS score & $15.0 \pm 26.4$ & $6.5 \pm 16.6$ & 0.168 \\
\hline WORC total & $366.2 \pm 551.5$ & $171.2 \pm 309.5$ & 0.116 \\
\hline Physical & $91.2 \pm 141.5$ & $44.8 \pm 76.6$ & 0.140 \\
\hline Sports & $86.2 \pm 129.2$ & $49.5 \pm 83.5$ & 0.227 \\
\hline Work & $83.5 \pm 126.4$ & $39.8 \pm 83.3$ & 0.147 \\
\hline Lifestyle & $58.7 \pm 97.0$ & $22.5 \pm 55.6$ & 0.100 \\
\hline Emotion & $46.5 \pm 82.8$ & $14.5 \pm 31.1$ & 0.060 \\
\hline \multicolumn{4}{|l|}{$\operatorname{ROM}\left(^{\circ}\right)$} \\
\hline Frontal & $168.5 \pm 26.0$ & $176.3 \pm 13.8$ & 0.172 \\
\hline Abduction & $166.7 \pm 28.6$ & $173.3 \pm 20.0$ & 0.343 \\
\hline
\end{tabular}

Values are presented as mean \pm standard deviation.

PRP: platelet-rich plasma, CS: corticosteroid, VAS: visual analog scale, WORC: Western Ontario Rotator Cuff Index, ROM: range of motion.

Table 3. Mean follow-up of the two groups' adverse events during the treatments and surgeries that excluded patients from further follow-up

\begin{tabular}{lccr}
\hline Variable & PRP group $(\mathrm{n}=35)$ & CS group $(\mathrm{n}=40)$ & $\mathrm{p}$-value \\
\hline No. of injections & $3.1 \pm 1.1$ & $1.1 \pm 0.3$ & $40(100.0)$ \\
Physical therapy & $35(100.0)$ & $40(100.0)$ & 1.000 \\
NSAIDs therapy & $35(100.0)$ & $33.6 \pm 16.3$ & 1.000 \\
Follow-up (mo) & $21.1 \pm 8.7$ & 0 & $<0.001$ \\
Adverse event & 0 & $11(27.5)$ & 1.000 \\
Arthroscopy & $7(20.0)$ & $11(27.5)$ & 0.589 \\
Shoulder surgery & $7(20.0)$ & & 0.589 \\
\hline
\end{tabular}

Values are presented as mean \pm standard deviation or number (\%).

PRP: platelet-rich plasma, CS: corticosteroid, NSAID: nonsteroidal anti-inflammatory drug. 


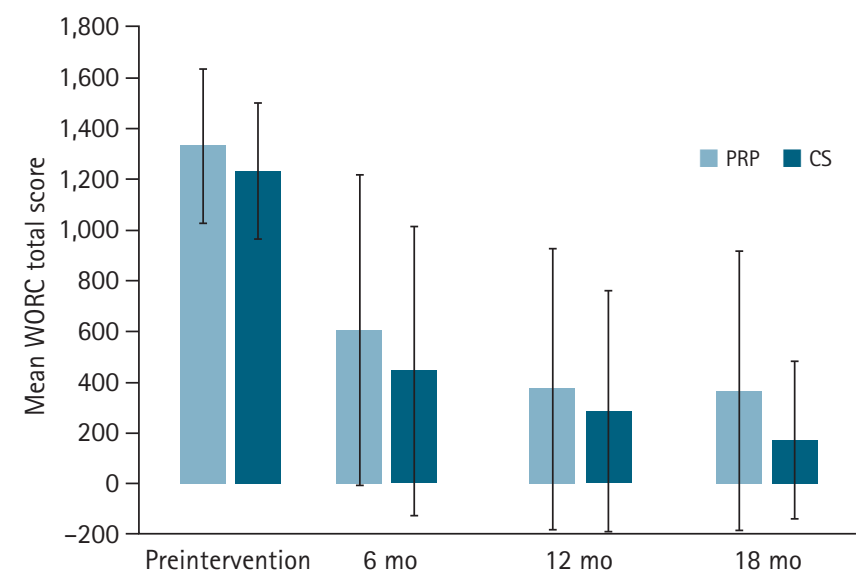

Fig. 3. Mean values of the Western Ontario Rotator Cuff Index (WORC) total score with 1 standard deviation (SD) during the follow-up between the corticosteroid (CS) group and the platelet-rich plasma (PRP) group. No significant differences were detected between the two groups.

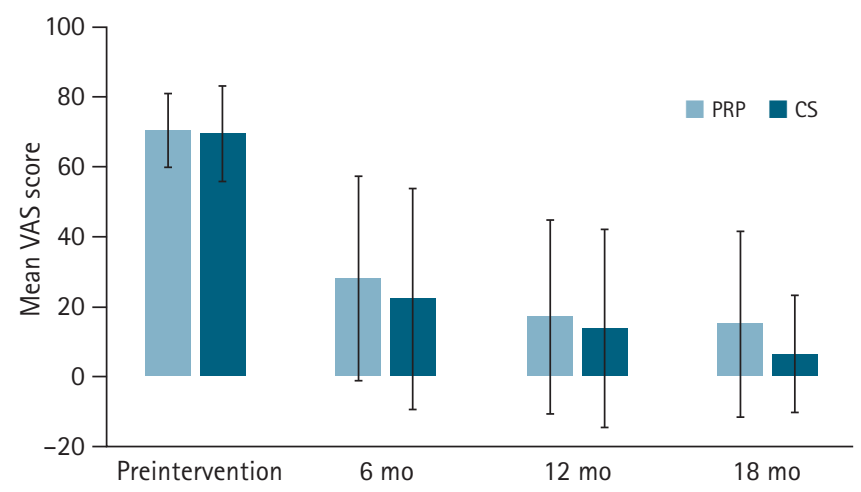

Fig. 4. Mean values of visual analog scale (VAS) for pain with 1 standard deviation during the follow-up between the corticosteroid (CS) group and the platelet-rich plasma (PRP) group. No significant differences were detected between the two groups.

found only three similarly conducted previous studies comparing subacromial injections of PRP to CS $[16,18,22]$. Among them, only Say et al. [17] reported CS to be superior to PRP in the treatment of subacromial impingement syndrome in their study of 60 patients. However, the study was not randomized, and the follow-up was short (6 months) [16]. Conversely, Shams et al. [19] demonstrated that the PRP group had better results in early stages of follow-up (3 months), but they detected no statistical differences in the long-term (6 months) results. Their study was randomized, including MRI for confirmed partial RC ruptures with persistent (over 3 months) shoulder pain, but only 40 patients were enrolled and documentation of detailed demographic data was not included [19]. Finally, von Wehren et al. [22] reported that there was earlier benefit favoring PRP in their study of 50 patients with partial RC tear, however no differences were detected at 6 months of follow-up [22]. Their limitations were the absence of randomization and relatively few patients [22]. Both Shams et al. [19] and von Wehren et al. [22] concluded that PRP might be a good alternative to subacromial CS injections.

The strengths of our study included a larger number of patients and a long follow-up in a comparative matter compared with the previously published studies. The mean follow-up was clearly longer than in previous studies, at over a year. Preintervention inclusion and exclusion criteria were strict and thorough with up to three imaging modalities involved and an experienced orthopedist to include only patients with RC tendinosis/tendinitis sometimes accompanied by subacromial bursitis or, rarely, a small/marginal tear in the RC tendon. Demographic and clinical data were meticulously collected.

It is also important to mention the concurrent physical therapy that both groups received; however, this is a necessary limitation as physical therapy is essential part of the treatment and rehabilitation in shoulder area diseases. It is important to note that injection therapies and analgesics may enable struggling patients to begin physical therapy, which may otherwise prove to be too difficult due to pain. Physical therapy may explain some of the symptom changes during follow up, but its impact is dramatically reduced since the same protocol was applied to both groups. The overall effect of paracetamol and NSAID is difficult to evaluate due to the lack of data concerning their total amounts. However, the prescribed amounts of paracetamol and NSAID could last for a maximum of 1 month. Since both groups had the same pain medication, this effect is likely to be similar in both groups during the early follow-up.

There were several limitations of our study including its retrospective design, lack of randomization, lack of placebo control, lack of rotational ROM data, and differences in comorbidities between the two groups-particularly the significant difference in the female to male sex ratio and higher WORC emotions sub score in the CS group (Fig. 2, Table 1). The use of anatomical landmarks instead of ultrasound can be considered a methodological limitation. We acknowledge that ultrasound-guided injections are more accurate than landmark-guided injections; however, there are studies showing no clinical difference between ultrasound-guided and landmark-guided injections [23,24].

Our study design might limit the interpretation and generalization of the results, but a previous study suggests that the placebo effect is only detectable within 1 month of follow-up [25]. This study primarily addresses the long-term results, and the relevance of a placebo control group is smaller in the long-term follow-up. The rotational ROM data were incomplete and therefore were not included in the analysis. This leaves an uncharted area 
in the clinical response to the treatments. Confounding factors of this study were higher female to male sex ratio in the PRP group, concurrent physical therapy in both groups, and higher baseline mean of comorbidities in the CS group than in the PRP group. Nonetheless, the PRP group included more females than CS group, which might explain the lower mean pretreatment WORC emotions subscore in the CS group as female patients usually report more symptoms than males [26].

There is also a possibility for information and selection bias. We analyzed more than one clinical score to accurately evaluate different aspects of function and symptoms to reduce potential information bias. The rationale for treating patients with PRP or CS was that the patients did not have any condition or disease that required surgical intervention at the time of treatment. Injection therapy was a further conservative treatment attempt with no specific patient selection protocol other than patients simply deciding whether to try injection therapy with PRP or with CS. This does not entirely remove possibility of selection bias, but it does reduce the impact. The injection protocol was inherently different, of course; previous data suggests that multiple PRP injections work better than a single PRP injection, while multiple CS injections in short succession may cause harm $[27,28]$

Although larger than previous studies, our sample size was relatively small, with a post hoc statistical power of $47.5 \%$. Up to seven patients (20\%) in the PRP group and 11 patients (27.5\%) in the CS group ended up receiving surgical treatment for their shoulder issues during the follow-up.

CS injections have higher risk for complications than PRP injections [28-30]. PRP may offer a valid alternative to CS considering that there are no documented significant adverse effects in PRP treatments, even in the long term [6,8,11,19,28-30]. Whenever symptoms return, PRP treatment can be repeated; conversely, multiple CS injections should be avoided. PRP treatments may be beneficial over CS treatments in patients who have not received symptom relief from previous CS treatments, have concerns for local or systemic CS effects, or are still considering possible operative treatment within 1 to 6 months, as prior CS injections increase the risk for surgery complications and revisions $[29,30]$. On the other hand, CS injections might work well for patients who are not good surgical candidates. Regardless of the injection therapy, concurrent physical therapy is still advised because of its proven benefits.

Further, larger randomized controlled trials are warranted to validate this promising treatment modality. Moreover, the role of PRP as a potential disease modifying agent is unclear, and adding imaging to the follow-up protocol would be beneficial. Given the outcomes of our study and despite the higher number of injections needed, we recommend considering PRP injection as a viable treatment option for RC tendinopathies because it has shown comparable effects to CS injections with no detected adverse effects.

\section{ORCID}

Salvatore Giordano

https://orcid.org/0000-0001-5981-6870

\section{REFERENCES}

1. Luime JJ, Koes BW, Hendriksen IJ, et al. Prevalence and incidence of shoulder pain in the general population: a systematic review. Scand J Rheumatol 2004;33:73-81.

2. Lewis J. Rotator cuff related shoulder pain: assessment, management and uncertainties. Man Ther 2016;23:57-68.

3. Diercks R, Bron C, Dorrestijn O, et al. Guideline for diagnosis and treatment of subacromial pain syndrome: a multidisciplinary review by the Dutch Orthopaedic Association. Acta Orthop 2014;85:314-22.

4. Ketola S, Lehtinen JT, Arnala I. Arthroscopic decompression not recommended in the treatment of rotator cuff tendinopathy: a final review of a randomised controlled trial at a minimum follow-up of ten years. Bone Joint J 2017;99:799-805.

5. Lin MT, Chiang CF, Wu CH, Huang YT, Tu YK, Wang TG. Comparative effectiveness of injection therapies in rotator cuff tendinopathy: a systematic review, pairwise and network meta-analysis of randomized controlled trials. Arch Phys Med Rehabil 2019;100:336-49. e15.

6. Chen X, Jones IA, Park C, Vangsness CT Jr. The efficacy of platelet-rich plasma on tendon and ligament healing: a systematic review and meta-analysis with bias assessment. Am J Sports Med 2018;46:2020-32.

7. Shen L, Yuan T, Chen S, Xie X, Zhang C. The temporal effect of platelet-rich plasma on pain and physical function in the treatment of knee osteoarthritis: systematic review and meta-analysis of randomized controlled trials. J Orthop Surg Res 2017;12: 16.

8. Desai VS, Camp CL, Boddapati V, Dines JS, Brockmeier SF, Werner BC. Increasing numbers of shoulder corticosteroid injections within a year preoperatively may be associated with a higher rate of subsequent revision rotator cuff surgery. Arthroscopy 2019;35:45-50.

9. Mohamadi A, Chan JJ, Claessen FM, Ring D, Chen NC. Corticosteroid injections give small and transient pain relief in rotator cuff tendinosis: a meta-analysis. Clin Orthop Relat Res 
2017;475:232-43.

10. Maman E, Yehuda C, Pritsch T, et al. Detrimental effect of repeated and single subacromial corticosteroid injections on the intact and injured rotator cuff: a biomechanical and imaging study in rats. Am J Sports Med 2016;44:177-82.

11. Freire V, Bureau NJ. Injectable corticosteroids: take precautions and use caution. Semin Musculoskelet Radiol 2016;20:401-8.

12. Zhang AZ, Ficklscherer A, Gülecyüz MF, et al. Cell toxicity in fibroblasts, tenocytes, and human mesenchymal stem cells: a comparison of necrosis and apoptosis-inducing ability in ropivacaine, bupivacaine, and triamcinolone. Arthroscopy 2017;33: 840-8.

13. Jo CH, Lee SY, Yoon KS, Oh S, Shin S. Allogenic pure platelet-rich plasma therapy for rotator cuff disease: a bench and bed study. Am J Sports Med 2018;46:3142-54.

14. Pauly S, Klatte-Schulz F, Stahnke K, Scheibel M, Wildemann B. The effect of autologous platelet rich plasma on tenocytes of the human rotator cuff. BMC Musculoskelet Disord 2018;19:422.

15. Kim SJ, Kim EK, Kim SJ, Song DH. Effects of bone marrow aspirate concentrate and platelet-rich plasma on patients with partial tear of the rotator cuff tendon. J Orthop Surg Res 2018; 13:1.

16. Nejati P, Ghahremaninia A, Naderi F, Gharibzadeh S, Mazaherinezhad A. Treatment of subacromial impingement syndrome: platelet-rich plasma or exercise therapy? A randomized controlled trial. Orthop J Sports Med 2017;5:2325967117702366.

17. Say F, Gurler D, Bulbul M. Platelet-rich plasma versus steroid injection for subacromial impingement syndrome. J Orthop Surg (Hong Kong) 2016;24:62-6.

18. Scarpone M, Rabago D, Snell E, et al. Effectiveness of platelet-rich plasma injection for rotator cuff tendinopathy: a prospective open-label study. Glob Adv Health Med 2013;2:26-31.

19. Shams A, El-Sayed M, Gamal O, Ewes W. Subacromial injection of autologous platelet-rich plasma versus corticosteroid for the treatment of symptomatic partial rotator cuff tears. Eur J Orthop Surg Traumatol 2016;26:837-42.

20. Hurley ET, Lim Fat D, Moran CJ, Mullett H. The efficacy of platelet-rich plasma and platelet-rich fibrin in arthroscopic rotator cuff repair: a meta-analysis of randomized controlled trials. Am J Sports Med 2019;47:753-61.

21. Randelli P, Arrigoni P, Ragone V, Aliprandi A, Cabitza P. Platelet rich plasma in arthroscopic rotator cuff repair: a prospective RCT study, 2-year follow-up. J Shoulder Elbow Surg 2011;20: 518-28.

22. von Wehren L, Blanke F, Todorov A, Heisterbach P, Sailer J, Majewski M. The effect of subacromial injections of autologous conditioned plasma versus cortisone for the treatment of symptomatic partial rotator cuff tears. Knee Surg Sports Traumatol Arthrosc 2016;24:3787-92.

23. Bhayana H, Mishra P, Tandon A, Pankaj A, Pandey R, Malhotra R. Ultrasound guided versus landmark guided corticosteroid injection in patients with rotator cuff syndrome: randomised controlled trial. J Clin Orthop Trauma 2018;9(Suppl 1):S80-5.

24. Cole BF, Peters KS, Hackett L, Murrell GA. Ultrasound-guided versus blind subacromial corticosteroid injections for subacromial impingement syndrome: a randomized, double-blind clinical trial. Am J Sports Med 2016;44:702-7.

25. Lin KY, Yang CC, Hsu CJ, Yeh ML, Renn JH. Intra-articular injection of platelet-rich plasma is superior to hyaluronic acid or saline solution in the treatment of mild to moderate knee osteoarthritis: a randomized, double-blind, triple-parallel, placebo-controlled clinical trial. Arthroscopy 2019;35:106-17.

26. Brown DA, Elsass JA, Miller AJ, Reed LE, Reneker JC. Differences in symptom reporting between males and females at baseline and after a sports-related concussion: a systematic review and meta-analysis. Sports Med 2015;45:1027-40.

27. Görmeli G, Görmeli CA, Ataoglu B, Çolak C, Aslantürk O, Ertem K. Multiple PRP injections are more effective than single injections and hyaluronic acid in knees with early osteoarthritis: a randomized, double-blind, placebo-controlled trial. Knee Surg Sports Traumatol Arthrosc 2017;25:958-65.

28. Ramírez JP, Bonati-Richardson F, García MP, et al. Intra-articular treatment with corticosteroids increases apoptosis in human rotator cuff tears. Connect Tissue Res 2019;60:283-90.

29. Lobo-Escolar L, Ramazzini-Castro R, Codina-Grañó D, Lobo E, Minguell-Monyart J, Ardèvol J. Risk factors for symptomatic retears after arthroscopic repair of full-thickness rotator cuff tears. J Shoulder Elbow Surg 2021;30:27-33.

30. Puzzitiello RN, Patel BH, Nwachukwu BU, Allen AA, Forsythe B, Salzler MJ. Adverse impact of corticosteroid injection on rotator cuff tendon health and repair: a systematic review. Arthroscopy 2020;36:1468-75. 